\title{
MONOUNARY ALGEBRAS WITH EASY DIRECT LIMITS
}

\author{
EMÍLIA HALUŠKOVÁ
}

Received 2015-09-21

\begin{abstract}
Let $\mathcal{A}$ be an algebra such that exactly algebras isomorphic to a retract of $\mathcal{A}$ can be constructed from $\mathcal{A}$ by direct limits. Then $\mathcal{A}$ is said to be an algebra with easy direct limits. We will prove that if $\mathcal{A}$ is a monounary, then $\mathcal{A}$ is countable and the number of retracts of $\mathcal{A}$ is not equal to $\aleph_{0}$. Further, we will see that the number of non-isomorphic monounary algebras with easy direct limits is $2^{\aleph_{0}}$.
\end{abstract}

2010 Mathematics Subject Classification: 08B25; 08A60; 03E17; 03C99; 40A05; 40J05

Keywords: algebra, direct limit, retract, term, monounary algebra

\section{INTRODUCTION}

The importance of the notion of retract is well known and commonly appreciated in mathematics. There are many papers dealing with retracts of algebraic structures, see e.g. [7], [10] for non-complete review. Actual results concerning monounary algebras can be found in [1],[9] and [8].

The algebraic construction of direct limit is a well-known method of building up new algebras from given ones. It helps to make progress in particular topics, recently see e.g. [11], [12]. Furthermore it is interesting to investigate this construction generally, cf.[6], [15], [14].

Let $\mathcal{A}$ be an algebra. We denote by $\underset{\mathbf{L}}{\rightarrow} \mathcal{A}$ the class of all isomorphic copies of direct limits which can be obtained from $\mathcal{A}$ and we denote by $\mathbf{R} \mathcal{A}$ the set of all retracts of $\mathcal{A}$. Then $\mathbf{R} \mathcal{A} \subseteq \underline{\mathbf{L}} \mathcal{A}$. We will say that $\mathcal{A}$ is an algebra with easy direct limits if every algebra from $\underset{\mathbf{L}}{\rightarrow} \vec{A}$ is isomorphic to a retract of $\mathcal{A}$.

The aim of this paper is to investigate algebras with easy direct limits. If $\mathcal{A}$ is finite, then $\mathcal{A}$ is an algebra with easy direct limits, cf.[5]. Some infinite algebras with easy direct limits can be found in [4].

We will prove results concerning monounary algebras. Properties of monounary algebras with easy direct limits run from section 4 . A monounary algebra with easy direct limits is countable. It contains a line or a cycle in every component. There

Supported by the grants VEGA 2/0028/13 and VEGA 2/0044/16. 
enter at most finitely many arrows to every point of it. The number of retracts of it is never equal to $\aleph_{0}$.

The section 5 contains a description of all monounary algebras with easy direct limits which have a bijective basic operation. This description implies that the number of non-isomorphic algebras with easy direct limits is equal to $2^{\boldsymbol{\aleph}_{0}}$, see Thm.3.

\section{PRELIMINARIES}

The cardinality of a set $A$ is denoted by $\|A\|$. The set of all positive integers is denoted by $\mathbb{N}$ and the set of all non-negative integers is denoted by $\mathbb{N}_{0}$.

We deal with monounary algebras. The fundamental operation is denoted by $f$. Short remarks about algebras of arbitrary type are before Lemma 6 and after Example 1 .

For monounary terminology see e.g. [10], [13].

Let $\mathcal{A}=(A, f)$ be a monounary algebra. A subset $B$ of $A$ is termed as a chain of $\mathcal{A}$ if for every $a, b \in B$ there is $n \in \mathbb{N}_{0}$ either $f^{n}(a)=b$ or $f^{n}(b)=a$. If $A$ is a chain of $\mathcal{A}$, then we will say that $\mathcal{A}$ is a basic algebra.

Basic monounary algebras were introduced in [7] and will be used in the proof of Cor 3 .

If $\mathcal{A}$ is connected and contains no cycle, then it is said to be a line, if for each $a \in A$ there exists an unique $b \in A$ with $f(b)=a$. A line is a basic algebra.

Denote

$$
\begin{gathered}
\downarrow b=\left\{d \in A: f^{k}(d)=b \text { for some } k \in \mathbb{N}_{0}\right\}, \\
f^{-1}(b)=\{d \in A: f(d)=b\}, \\
\uparrow b=\left\{f^{k}(b): k \in \mathbb{N}_{0}\right\} .
\end{gathered}
$$

We remind the notion of degree $s(a)$ of an element $a \in A$. Let us define by $A^{(\infty)}$ the set of all elements $a \in A$ such that the set $\downarrow a$ contains an infinite chain of $\mathcal{A}$. Further, we put $A^{(0)}=\left\{a \in A: f^{-1}(a)=\varnothing\right\}$. Now we define a set $A^{(\lambda)} \subseteq A$ for each ordinal $\lambda$ by induction. Assume that we have defined $A^{(\alpha)}$ for each ordinal $\alpha<\lambda$. Then we put

$$
A^{(\lambda)}=\left\{a \in A \backslash \bigcup_{\alpha<\lambda} A^{(\alpha)}: f^{-1}(a) \subseteq \bigcup_{\alpha<\lambda} A^{(\alpha)}\right\} .
$$

The sets $A^{(\lambda)}$ are pairwise disjoint. For each $a \in A$, either $a \in A^{(\infty)}$ or there is an ordinal $\lambda$ with $a \in A^{(\lambda)}$. In the former case we put $s(a)=\infty$, in the latter we set $s(a)=\lambda$.

Let $\mathscr{B}=(B, f)$ be a subalgebra of $\mathcal{A}$. Then $\mathscr{B}$ is said to be a retract of $\mathcal{A}$ if there exists an endomorphism $h$ of $\mathcal{A}$ such that $h(A)=B$ and $h^{2}(a)=h(a)$ for every $a \in A$.

We will say that $\mathscr{B}$ is a basic retract of $\mathcal{A}$, if $\mathscr{B}$ is a retract of $\mathcal{A}$ and $\mathscr{B}$ is a basic algebra. 
The notion of a direct limit we apply by [2], $\$ 21$.

Let $\langle P, \leq\rangle$ be a directed partially ordered set. For each $p \in P$, let $\mathcal{A}_{p}=\left(A_{p}, f\right)$ be a monounary algebra. Assume that if $p, q \in P, p \neq q$, then $A_{p} \cap A_{q}=\varnothing$. Suppose that for each pair of elements $p$ and $q$ in $P$ with $p<q$, we have a homomorphism $\varphi_{p q}$ of $\mathscr{A}_{p}$ into $\mathscr{A}_{q}$ such that $p<q<s$ implies that $\varphi_{p s}=\varphi_{p q} \circ \varphi_{q s}$. For each $p \in P$, suppose that $\varphi_{p p}$ is the identity on $A_{p}$. The family $\left\{P, \mathcal{A}_{p}, \varphi_{p q}\right\}$ is said to be direct.

Assume that $p, q \in P$ and $a \in A_{p}, b \in A_{q}$. Put $a \equiv b$ if there exists $s \in P$ with $p \leq s, q \leq s$ such that $\varphi_{p s}(a)=\varphi_{q s}(b)$.

$$
\begin{aligned}
& \text { For each } a \in \bigcup_{p \in P} A_{p} \text { put } \bar{a}=\left\{b \in \bigcup_{p \in P} A_{p}: a \equiv b\right\} . \\
& \text { Denote } \bar{A}=\left\{\bar{a}: a \in \bigcup_{p \in P} A_{p}\right\} . \text { Define } f(\bar{a})=\overline{f(a)} .
\end{aligned}
$$

Then the monounary algebra $\bar{A}=(\bar{A}, f)$ is said to be a direct limit of the direct family $\left\{P, \mathcal{A}_{p}, \varphi_{p q}\right\}$. We express this situation as follows

$$
\left\{P, \mathcal{A}_{p}, \varphi_{p q}\right\} \longrightarrow \overline{\mathcal{A}} \text {. }
$$

Note that in the category theory this construction corresponds to (directed) colimit.

Let (1) be valid. The following lemmas follow from the definition of the direct limit.

Lemma 1. Let $p \in P$ and $a, b \in A_{p}$. If $a, b$ are in one component of $\mathcal{A}_{p}$, then $\bar{a}, \bar{b}$ are in one component of $\overline{\mathcal{A}}$.

Lemma 2. Let $p \in P$ and $a \in A_{p}$. If $a$ is a cyclic element of $\mathcal{A}_{p}$, then $\bar{a}$ is a cyclic element of $\overline{\mathcal{A}}$.

Lemma 3. Let $u \in \bar{A}$ belong to an $n$-element cycle of $\overline{\mathcal{A}}$. Then there exist $p \in P$ and $a \in A_{p} \cap u$ such that a belongs to an n-element cycle of $\mathcal{A}_{p}$. Moreover, for all $q \geq p$ the point $\varphi_{p q}(a)$ belongs to an $n$-element cycle of $\mathcal{A}_{q}$.

Lemma 4. Let $u_{1}, \ldots, u_{k} \in \bar{A}$. Then there exist $p \in P$ and $a_{1}, \ldots, a_{k} \in A_{p}$ such that $a_{1} \in u_{1}, \ldots, a_{k} \in u_{k}$.

Lemma 5. Let the operation $f$ be injective (surjective) on $A_{p}$ for all $p \in P$. Then the operation $f$ is injective (surjective) on $\bar{A}$.

Let $\mathcal{A}$ be an algebra. The class $\underset{\mathbf{L}}{\rightarrow} \mathcal{A}$ is created by all direct limits of families in which only algebras isomorphic to $\overrightarrow{\mathcal{A}}$ occur.

The following lemma will be repeatly used in this paper. It holds for algebras of arbitrary type and it is proved as Lemma 2 of [4]:

Lemma 6. If $\mathcal{A}^{\prime} \in \mathbf{R} \mathcal{A}$ and $\mathscr{B} \in \underset{\mathbf{L}}{\rightarrow} \mathcal{A}^{\prime}$, then $\mathscr{B} \in \underset{\mathbf{L}}{\rightarrow} \mathcal{A}$. 
Let $I$ be a nonempty set. For each $i \in I$ let $\mathcal{B}_{i}=\left(B_{i}, f\right)$ be a monounary algebra. We denote by $\sum_{i \in I} \mathscr{B}_{i}$ a monounary algebra which is a disjoint union of algebras $\mathscr{B}_{i}, i \in I$.

Let $\mathcal{A}=\sum_{i \in I} \mathscr{B}_{i}$ and $\mathscr{B}_{i}$ be connected for all $i \in I$. Let $i \in I$. If $\mathscr{B}_{i}$ contains a cycle of length $k$ for some $k \in \mathbb{N}$, then we denote by $\ell_{i}$ a cycle of length $k$. Else we denote by $\ell_{i}$ a line. Put $\mathcal{A}^{\diamond}=\sum_{i \in I} \ell_{i}$.

The following lemma is proved as Lemma 4 in [3].

Lemma 7. $\mathcal{A}^{\diamond} \in \underset{\mathbf{L}}{\rightarrow} \mathcal{A}$

Corollary 1. Let $\mathcal{A}=(A, f)$ be a monounary algebra. Then there exists $\mathscr{B} \in \underset{\mathbf{L}}{\rightarrow} \mathcal{A}$ such that the operation of $\mathcal{B}$ is bijective.

\section{Auxiliary RESUlts}

In this section let $\mathcal{A}=(A, f)$ be a monounary algebra. We will prove five preliminary lemmas about algebras in $\underset{\mathbf{L}}{\rightarrow} \mathcal{A}$.

Lemma 8. Let $\mathfrak{D}=(D, f)$ be a component of $\mathcal{A}=(A, f)$ and $v$ be an infinite cardinal number. If $\mathcal{A}$ has $v$ components isomorphic to $\mathscr{D}$, then $\underset{\mathbf{L}}{\rightarrow} \mathcal{A}$ contains an algebra with more than $v$ components isomorphic to $\mathfrak{D}$.

Proof. Let $\mu$ be the cardinality of $D$. Denote by $\mathcal{B}=(B, f)$ a subalgebra of $\mathcal{A}$ which is created by $v$ components isomorphic to $\mathscr{D}$. Take $\kappa>v$ and $K$ the set of cardinality $\kappa$. Let $i \in K$. Let $B_{i}$ be a set of the cardinality $\nu \cdot \mu, B_{i} \cap B_{j}=\varnothing$ for $i \neq j$. Let $f$ be defined on $B_{i}$ in a such way that $\left(B_{i}, f\right) \cong \mathscr{B}$.

Take $P$ the set of all finite subsets of $K$. Consider $\langle P, \subseteq\rangle$. Let $p \in P$. Put

$$
\begin{gathered}
A_{p}=\left\{(d, p): d \in \bigcup_{j \in p} B_{j} \cup(A \backslash B)\right\}, \\
f(d, p)=(f(d), p), \\
\mathcal{A}_{p}=\left(A_{p}, f\right) .
\end{gathered}
$$

We have $\left(\bigcup_{j \in p} B_{j}, f\right) \cong \mathscr{B}$ since $p$ is finite. Therefore $\mathcal{A}_{p} \cong \mathcal{A}$.

Let $p, q \in P, p \subseteq q$ and $\varphi_{p q}(x, p)=(x, q)$ for every $(x, p) \in A_{p}$. Obviously $\left\{P, \mathcal{A}_{p}, \varphi_{p q}\right\}$ is a direct family.

Let $\overline{\mathscr{A}}=(\bar{A}, f)$ be a direct limit of this family. Then $\overline{\mathscr{A}} \in \underset{\mathbf{L}}{\rightarrow} \mathcal{A}$. If $i, j \in K, i \neq j$ and $d_{i} \in B_{i}, d_{j} \in B_{j}$, then $\overline{\left(d_{i},\{i\}\right)}$ and $\overline{\left(d_{j},\{j\}\right)}$ are in isomorphic but different components of $\overline{\mathcal{A}}$. Therefore $\overline{\mathcal{A}}$ contains at least $\kappa>v$ components isomorphic to D.

Lemma 9. Let $\kappa$ be an infinite cardinal. Let $a \in A$ and $D \subseteq A$ be such that

(1) $D \subseteq f^{-1}(a)$;

(2) $\left(\downarrow \bar{d}_{1} \cup \uparrow a, f\right) \cong\left(\downarrow d_{2} \cup \uparrow a, f\right)$ for all $d_{1}, d_{2} \in D$; 
(3) $D$ is infinite.

Then there exists $\overline{\mathscr{A}}=(\bar{A}, f) \in \underset{\mathbf{L}}{\rightarrow} \mathcal{A}$ such that $\|\bar{A}\|>\kappa$.

Proof. Denote $B=\bigcup_{d \in D} \downarrow d$ and $v=\|B\|$. Let $M$ be a set of cardinality greater than $\kappa$. For $m \in M$ let $B_{m}$ be a set of cardinality of $\nu$ and $D_{m}$ be such that $D_{m} \subseteq B_{m}$ and $\left\|D_{m}\right\|=\|D\|$. Assume that $B_{m} \cap A=\varnothing$ and $B_{m} \cap B_{m^{\prime}}=\varnothing$ for $m \neq m^{\prime}$. Further, let $f$ be defined on $B_{m}$ in a such way that $f(t)=a$ for $t \in D_{m}$ and $\left(B_{m} \cup \uparrow\right.$ $a, f) \cong(B \cup \uparrow a, f)$.

Let $P$ be the set of all finite subsets of $M$. Let $p \in P$. Put

$$
A_{p}=\left\{(d, p): d \in \bigcup_{m \in p} B_{m} \cup(A \backslash B)\right\}, f(d, p)=(f(d), p) .
$$

Let $\mathcal{A}_{p}=\left(A_{p}, f\right)$. Since $p$ is finite, we have that $\mathcal{A}_{p} \cong \mathcal{A}$.

Let $p, q \in P, p \subseteq q$. We define $\varphi_{p q}(x, p)=(x, q)$ for every $(x, p) \in A_{p}$. We have that $\varphi_{p q}$ is a homomorphism from $\mathcal{A}_{p}$ into $\mathcal{A}_{q}$. Thus $\left\{P, \mathcal{A}_{p}, \varphi_{p q}\right\}$ is a direct family. Let $\overline{\mathcal{A}}=(\bar{A}, f)$ be a direct limit of this family.

Suppose that $v \in \bar{A}$. Let $(d, p) \in v \cap A_{p}$. If $d \in A \backslash B$, then $v=\{(d, q): q \in P\}$. If $d \in B_{m}$ for some $m \in p$, then $v=\{(d, q): q \in\{s \in P: m \in s\}\}=\overline{(d,\{m\})}$. Further, $\overline{(d,\{m\})} \neq \overline{(t,\{n\})}$ for every $d, t \in \cup_{m \in M} D_{m}, d \neq t$ and $m, n \in M$.

Take

$$
V=\left\{\overline{(t,\{m\})}: t \in D_{m}, m \in M\right\} .
$$

We obtain

$$
\|\bar{A}\| \geq\|V\|=\sum_{m \in M}\left\|D_{m}\right\|=\|M\| \cdot\|D\| \geq\|M\|>\kappa .
$$

Lemma 10. Let $a \in A$ be such that the set $K=\left\{d \in f^{-1}(a): s(d)=\infty\right\}$ is infinite. Then there exists $\overline{\mathscr{A}}=(\bar{A}, f) \in \underset{\mathbf{L}}{\rightarrow} \mathcal{A}$ such that $\|\bar{A}\|>\|A\|$.

Proof. Let $\mathcal{A}^{\prime}=\left(A^{\prime}, f\right) \in \mathbf{R} \mathcal{A}$ be such that $a \in A^{\prime}, f^{-1}(a) \cap A^{\prime}=K$ and $f$ is injective on $\downarrow d$ for all $d \in K$. The algebra $\mathcal{A}^{\prime}$ satisfies assumptions of Lemma 9 for the element $a$ and the set $K$. Take $\kappa=\|A\|$. Then there exists $\overline{\mathscr{A}}=(\bar{A}, f) \in \underline{\mathbf{L}} \mathcal{A}^{\prime}$ such that $\|\bar{A}\|>\|A\|$. We obtain $\overline{\mathscr{A}} \in \underset{\mathbf{L}}{\mathbf{L}} \mathcal{A}$ according to Lemma 6 .

Lemma 11. Let $k \in \mathbb{N}$ and $a \in A$ be such that the set $\left\{d \in f^{-1}(a): s(d) \leq k\right\}$ is infinite. Then there exists $\overline{\mathcal{A}}=(\bar{A}, f) \in \underset{\rightarrow}{\mathbf{L}} \mathcal{A}$ such that $\|\bar{A}\|>\|A\|$.

Proof. Denote $L=\left\{d \in f^{-1}(a): s(d) \leq k\right\}$. The elements of $L$ have degrees from the set $\{0,1, \ldots, k\}$. The infiniteness of $L$ implies that there exists $i \in\{0,1, \ldots, k\}$ such that the set $L$ contains $\|L\|$ elements of degree $i$.

There exists $\mathcal{A}^{\prime}=\left(A^{\prime}, f\right) \in \mathbf{R} \mathcal{A}$ such that $a \in A^{\prime}, f^{-1}(a) \cap A^{\prime}=f^{-1}(a)$ and $f$ is injective on $\downarrow d \cap A^{\prime}$ for all $d \in L$ such that $s(d)=i$. 
The algebra $\mathcal{A}^{\prime}$ satisfies assumptions of Lemma 9 for the element $a$ and the set $D=\left\{d \in f^{-1}(a): s(d)=i\right\}$. Take $\kappa=\|A\|$. Then there exists $\overline{\mathcal{A}}=(\bar{A}, f) \in \underset{\mathbf{L}}{\rightarrow} \mathcal{A}^{\prime}$ such that $\|\bar{A}\|>\|A\|$. We obtain $\overline{\mathcal{A}} \in \underset{\rightarrow}{\mathbf{L}} \mathcal{A}$ according to Lemma 6 .

Lemma 12. Let $a \in A$ and the sequence $\left\{d_{k}, k \in \mathbb{N}\right\}$ be such that $d_{k} \in f^{-1}(a)$, $s\left(d_{k}\right) \in \mathbb{N}$ and $s\left(d_{k}\right)<s\left(d_{k+1}\right)$ for every $k \in \mathbb{N}$. Then there exist $\overline{\mathcal{A}}=(\bar{A}, f) \in \underset{\mathbf{L}}{\rightarrow} \mathcal{A}$ and $u \in \bar{A}$ such that the set $\left\{v \in f^{-1}(u): s(v)=\infty\right\}$ is infinite

Proof. Let $\mathcal{A}^{\prime}=\left(A^{\prime}, f\right) \in \mathbf{R} \mathcal{A}$ be such that $a \in A^{\prime}, f^{-1}(a) \cap A^{\prime}=f^{-1}(a)$ and $f$ is injective on $\downarrow d_{k} \cap A^{\prime}$ for all $k \in \mathbb{N}$. Put

$$
D=\bigcup_{k \in \mathbb{N}}\left(\downarrow d_{k} \cap A^{\prime}\right) .
$$

Let $a^{\prime} \in D$. Then $f^{n}\left(a^{\prime}\right)=d_{k}$ for some uniquely determined $k \in \mathbb{N}$ and $n \in \mathbb{N}_{0}$, since $f$ is injective on $\downarrow d_{k} \cap A^{\prime}$. We will write $f^{-n}\left(d_{k}\right)=a^{\prime}$.

Let $p \in \mathbb{N}$. Put $A_{p}=\left\{(d, p): d \in A^{\prime}\right\}, f(d, p)=(f(d), p), \mathcal{A}_{p}=\left(A_{p}, f\right)$. Let $\varphi_{p, p+1}$ be a mapping from $A_{p}$ into $A_{p+1}$ such that $\varphi_{p, p+1}(d, p)=(d, p+1)$ for $d \in A^{\prime} \backslash D$ and $\varphi_{p, p+1}\left(f^{-n}\left(d_{k}\right), p\right)=\left(f^{-n}\left(d_{k+1}\right), p+1\right)$. This mapping is the homomorphism from $\mathcal{A}_{p}$ into $\mathcal{A}_{p+1}$. Consider a common linear order on $\mathbb{N}$. We obtained a direct family $\left\{\mathbb{N}, \mathcal{A}_{p}, \varphi_{p q}\right\}$. Let $\overline{\mathcal{A}}=(\bar{A}, f)$ be a direct limit of this family.

Take $u=\overline{(a, 1)}$. Then $u=\{(a, p): p \in \mathbb{N}\}$ and

$$
f^{-1}(u)=\left\{\overline{\left(d_{i}, 1\right)}, \overline{\left(d_{1}, i\right)}: i \in \mathbb{N}\right\} \cup\left\{\overline{(d, 1)}: d \in f^{-1}(a) \backslash\left\{d_{k}, k \in \mathbb{N}\right\}\right\} .
$$

Further, if $i \in \mathbb{N}$, then

$$
\begin{gathered}
\downarrow \overline{\left(d_{1}, i\right)}=\left\{\overline{\left(d_{1}, i\right)}, \overline{\left(f^{-1}\left(d_{1}\right), i\right)}, \ldots, \overline{\left(f^{-s\left(d_{1}\right)}\left(d_{1}\right), i\right)}, \overline{\left(f^{-s\left(d_{1}\right)-1}\left(d_{2}\right), i+1\right)},\right. \\
\left.\ldots, \overline{\left(f^{-s\left(d_{2}\right)}\left(d_{2}\right), i+1\right)}, \overline{\left(f^{-s\left(d_{2}\right)-1}\left(d_{3}\right), i+2\right)}, \ldots\right\}
\end{gathered}
$$

and $f$ is injective on $\downarrow \overline{\left(d_{1}, i\right)}$. Thus $s\left(\overline{\left(d_{1}, i\right)}\right)=\infty$. The set $\left\{\overline{\left(d_{1}, i\right)}: i \in \mathbb{N}\right\}$ is infinite, because $\varphi_{i, k}\left(d_{1}, i\right)=\left(d_{1+k-i}, k\right)$.

\section{THE MAIN RESULT}

Several properties of monounary algebras with easy direct limits are proved in this section. They do not give a full description of all monounary algebras with easy direct limits but allow to observe some interesting cardinal characteristics, see Cor 2 and Cor. 3. Prop. 1 can be generalized to universal algebras and this generalization is formulated in the end of this section. Other assertions are closely connected to an unary fundamental operation.

We will suppose that $\mathcal{A}=(A, f)$ is an algebra with easy direct limits in propositions $1-3$.

Proposition 1. There exists $\mathscr{B}=(B, f) \in \mathbf{R} \mathcal{A}$ such that $f$ is bijective on $B$. 
Proof. It follows from Cor. 1.

Proposition 2. The number of components of $\mathcal{A}$ without a cycle is finite and the number of components of $\mathcal{A}$ which contain an $n$-element cycle is finite for every $n \in \mathbb{N}$.

Proof. Let $\mathcal{A}=\sum_{k \in K} \mathscr{B}_{k}$, where $\mathscr{B}_{k}$ are connected for all $k \in K$.

Let $n \in \mathbb{N}$. Suppose that $\mathcal{A}$ contains infinitely many $n$-element cycles. Denote

$$
K^{\prime}=\left\{k \in K: \mathscr{B}_{k} \text { has a cycle of length } n\right\} .
$$

Let $k \in K$. Consider an algebra $\mathscr{D}_{k}$ such that $\mathscr{D}_{k}=\mathscr{B}_{k}$ if $k \notin K^{\prime}$ and $\mathscr{D}_{k}$ a cycle of length $n$ if $k \in K^{\prime}$. Take $\mathscr{D}=\sum_{k \in K} \mathscr{D}_{k}$.

We have $\mathscr{D} \in \mathbf{R} \mathcal{A}$. There exists $\mathscr{E} \in \underset{\mathbf{L}}{\rightarrow} \mathscr{D}$ such that $\mathscr{E}$ contains more cycles of length $n$ than $\mathscr{D}$ in view of Lemma 8. According to the number of $n$-element cycles of $\mathcal{D}$ we obtain that $\mathcal{E}$ is not isomorphic to a subalgebra of $\mathcal{A}$. That means $\mathcal{E}$ is not isomorphic to a retract of $\mathcal{A}$. In view of Lemma 6 we have $\mathcal{E} \in \mathbf{L} \rightarrow \mathcal{A}$.

Analogously we prove that $\mathcal{A}$ is not an algebra with easy direct limits if $\mathcal{A}$ contains infinitely many components with a line.

Finally, suppose that $\mathcal{A}$ contains infinitely many components which contain no cycle and no line. In view of Lemma 7 there exists an algebra of $\underset{\mathbf{L}}{\rightarrow} \mathcal{A}$ such that it contains an infinitely many components with a line. Therefore $\mathcal{A}$ is not an algebra with easy direct limits.

Proposition 3. The set $f^{-1}(a)$ is finite for every $a \in A$.

Proof. We denote $K_{a}=\left\{b \in f^{-1}(a): s(b)=\infty\right\}$ for every $a \in A$.

Assume that there exists $a \in A$ such that $K_{a}$ is infinite. We use Lemma 10 to obtain an algebra $\overline{\mathcal{A}}=(\bar{A}, f) \in \underset{\mathbf{L}}{\rightarrow} \mathcal{A}$ which has the cardinality greater than the set $A$. Thus $\overline{\mathscr{A}}$ is not isomorphic to a subalgebra of $\mathcal{A}$ and $\mathcal{A}$ is not an algebra with easy direct limits, a contradiction.

Now suppose that $a \in A$ is such that the set $f^{-1}(a)$ is infinite. Then $\left\{c \in f^{-1}(a)\right.$ : $s(c) \neq \infty\}$ is infinite. The assumption of Lemma 12 fails to hold, otherwise there is $\overline{\mathcal{A}} \in \underset{\mathbf{L}}{\rightarrow} \mathcal{A}$ such that $\overline{\mathcal{A}}$ is not a retract of $\mathcal{A}$, which is a contradiction. Therefore there exists $b \in f^{-1}(a)$ with $s(b)=\max \left\{s(c): c \in f^{-1}(a): s(c) \neq \infty\right\}$. This implies that either $s(a)=\infty$ or $\infty \neq s(a)=s(b)+1$.

If $s(a) \notin \mathbb{N} \cup\{\infty\}$, then $s(b) \geq \omega$ (where $\omega$ is the least infinite ordinal number), $b$ fulfils the assumption of Lemma 12 and this yields a contradiction too.

Further, let $s(a) \in \mathbb{N} \cup\{\infty\}$. Then $s(b) \in \mathbb{N}$ and $\left\{c \in f^{-1}(a): s(c) \neq \infty\right\}=\{c \in$ $\left.f^{-1}(a): s(c) \in \mathbb{N}\right\}=\left\{c \in f^{-1}(a): s(c) \leq s(b)\right\}$. According to Lemma 11 again there exists $\overline{\mathscr{A}} \in \underset{\mathbf{L}}{\rightarrow} \mathcal{A}$ such that $\overline{\mathscr{A}}$ is not a retract of $\mathcal{A}$, which is a contradiction.

Theorem 1. If $\mathcal{A}=(A, f)$ is an algebra with easy direct limits, then

(i) every component of $\mathcal{A}$ without a cycle contains a line, 


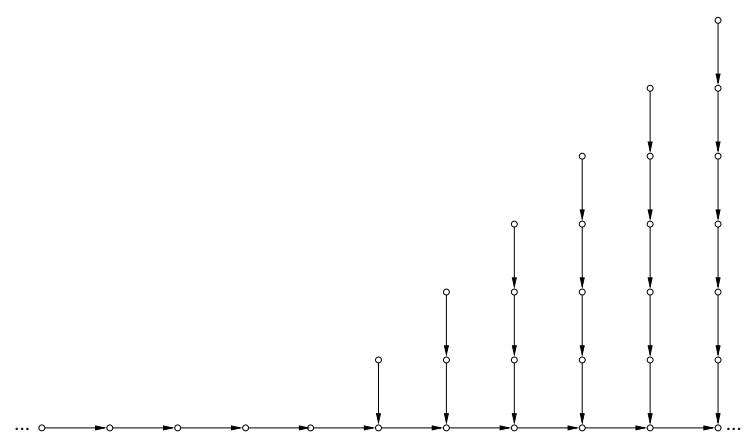

FIGURE 1. The algebra $\mathcal{A}$

(ii) the number of components of $\mathcal{A}$ without a cycle is finite,

(iii) the number of components of $\mathcal{A}$ which contain an n-element cycle is finite for every $n \in \mathbb{N}$,

(iv) the set $f^{-1}(a)$ is finite for every $a \in A$.

Proof. Properties (ii) and (iii) are valid according to Prop. 2. Therefore the number of lines of the algebra $\mathcal{A}^{\diamond}$ is finite. This yields that $\mathscr{A}^{\diamond}$ is isomorphic to a retract of $\mathcal{A}$ according to Lemma 7. Thus (i) is satisfied. Property (iv) is proved in Prop. 3.

It is easy to see that conditions (i) - (iv) are independent.

Corollary 2. If $\mathcal{A}=(A, f)$ is an algebra with easy direct limits, then $A$ is countable.

Proof. The number of components of $\mathcal{A}$ is countable according to (ii) and (iii). The number of elements of an arbitrary component of $\mathcal{A}$ is countable according to (iv).

Corollary 3. Let $\mathcal{A}=(A, f)$ be an algebra with easy direct limits. Then $\|\mathbf{R} \mathcal{A}\| \neq$ $\aleph_{0}$

Proof. We have $\|\mathbf{R} \mathcal{A}\| \leq 2^{\aleph_{0}}$ since $A$ is countable.

Every component of $\mathcal{A}$ contains a cycle or a line in view of Thm. 1 (i). Thus every component of $\mathcal{A}$ contains a basic retract. Therefore there is no component of $\mathcal{A}$ with the number of retracts equal to $\aleph_{0}$ according to Thm.5.9 of [7]. It implies $\|\mathbf{R} \mathcal{A}\| \neq \aleph_{0}$ according to Thm.5.10 of [7].

Example 1. Let

$$
A=\{(0,0),(a, 0),(-a, 0),(a, b): a, b \in \mathbb{N}, b \leq a\} .
$$

Let $f(a, b)=(a, b-1)$ for $b>1$ and $f(a, 0)=(a+1,0)$. Then $\mathcal{A}=(A, f)$ has properties (i)-(iv) and it is not an algebra with easy direct limits. 


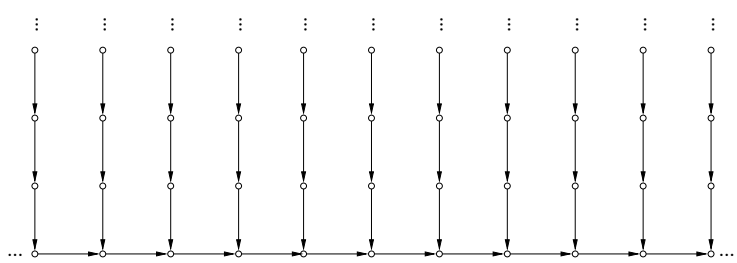

FIGURE 2. The algebra $\overline{\mathcal{A}}$

We hint why $\mathcal{A}$, see Fig.1, is not an algebra with easy direct limits. Consider $d \in A$ such that

1. $s(d)=\infty$,

2. $f$ is injective on $\downarrow d$,

3. there is $e \in A \backslash\{d\}$ such that $f(e)=f(d)$.

The algebra $\mathcal{A}$ contains exactly one point $d \in A$ which satisfies the conditions 1 . - 3, namely $d=(0,0)$.

For $n \in \mathbb{N}$ let $A_{n}=\{(a, b, n):(a, b) \in A\}$ and $f(a, b, n)=(f(a, b), n)$. Put $\mathcal{A}_{n}=$ $\left(A_{n}, f\right)$. Further, let $\varphi_{n, n+1}(a, b, n)=(a+1, b, n+1)$. Then $\left\{\mathbb{N}, \mathcal{A}_{n}, \varphi_{n m}\right\}$ is a direct family, $\mathcal{A}_{n} \cong \mathcal{A}$. Let $\left\{\mathbb{N}, \mathcal{A}_{n}, \varphi_{n m}\right\} \longrightarrow \overline{\mathcal{A}}$, see Fig.2.

For every $a>0$ we have as on Fig.3

$$
\begin{gathered}
f(\overline{(a, a, n+1)})=\overline{f(a, a, n+1)}=\overline{(f(a, a), n+1)}= \\
\overline{(a, a-1, n+1)}=\overline{\varphi_{n, n+1}(a-1, a-1, n)}=\overline{(a-1, a-1, n) .}
\end{gathered}
$$

Further,

$$
\overline{(1,1, n)} \neq \overline{(0,0, n)} \text { and } f(\overline{(1,1, n)})=\overline{(0,0, n-1)}=f(\overline{(0,0, n)}) \text {. }
$$

The elements $\overline{(1,1, n)}$ of $\overline{\mathcal{A}}$ for every $n \in \mathbb{N}$ are pairwise different elements which satisfy conditions $1 .-3$.

Remark 1. Corollary 1 and Proposition 1 can be generalized for universal algebras by the following way:

Let $\mathcal{A}$ be an algebra of type $F$ and $f$ be an unary term operation over $F$ such that $f$ is an endomorphism of the algebra $\mathcal{A}$. Then

(1) there exists $\mathscr{B}=(B, F) \in \underline{\mathbf{L}} \mathcal{A}$ such that $f$ is bijective on $B$,

(2) if $\mathcal{A}$ is an algebra with easy direct limits, then there exists $\mathscr{B}=(B, F) \in \mathbf{R} \mathcal{A}$ such that $f$ is bijective on $B$.

\section{Algebras with a bijective operation}

Let $A$ be a nonempty set and $f$ be a bijective operation from $A$ into $A$. We will prove that $\mathcal{A}=(A, f)$ is an algebra with easy direct limits if and only if the number of pairwise isomorphic components of $\mathcal{A}$ is finite. 

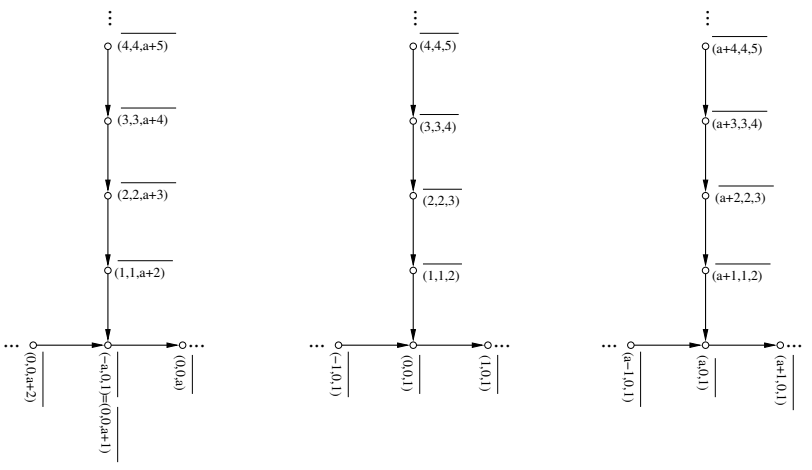

FIGURE 3. A description of $\overline{\mathcal{A}}, a \in \mathbb{N}$

Example 2. Let $\mathcal{A}=(A, f)$ be monounary algebra such that every component of $\mathcal{A}$ is a cycle of the length $2^{n}$ for some $n \in \mathbb{N}$ and for every $n \in \mathbb{N}$ there is exactly one cycle of the length $2^{n}$. Then $\mathcal{A}$ is an algebra with easy direct limts such that $A$ is countable and $\mathbf{R} \mathcal{A}$ consists of uncountable many non-isomorphic algebras. ( $\mathcal{A}$ is an algebra with easy direct limits according to the fact that every algebra of $\underset{\mathbf{L}}{\rightarrow} \mathcal{A}$ contains a 2-element cycle and by Lemmas 2 and 3.)

The following assertion is obvious.

Lemma 13. Let $\mathcal{A}=(A, f)$ be a monounary algebra. The following conditions are equivalent:

(i) the operation $f$ is bijective;

(ii) card $f^{-1}(a)=1$ for every $a \in A$;

(iii) if $\mathscr{B}$ is a component of $\mathcal{A}$, then $\mathscr{B}$ is a cycle or a line.

Theorem 2. Let $\mathcal{A}=(A, f)$ be a monounary algebra with a bijective operation. Then the following conditions are equivalent:

(a) A is an algebra with easy direct limits.

(b) The number of pairwise isomorphic components of $\mathcal{A}$ is finite.

(c) Conditions (i) - (iv) from Thm.1 are satisfied.

Proof. The condition (a) implies (b) according to Lemma 8.

Conditions (i) and (iv) from Thm.1 are satisfied since $\mathcal{A}$ has a bijective operation. Thus (b) is equivalent to (c).

Suppose that (b) is satisfied. Let (1) be valid and $\mathcal{A}_{p} \cong \mathcal{A}$. Then the operation $f$ of $\overline{\mathcal{A}}$ is bijective by Lemma 5 .

Let $\mathscr{B}_{1}, \ldots, \mathscr{B}_{k}$ be pairwise isomorphic components of $\overline{\mathcal{A}}$. Take $u_{i}$ an element of $\mathscr{B}_{i}$ for $i=1, \ldots, k$. According to Lemma 4 choose $p \in P$ and $a_{1}, \ldots, a_{k} \in A_{p}$ such 
that $a_{1} \in u_{1}, \ldots, a_{k} \in u_{k}$. Lemma 1 yields that $a_{1}, \ldots, a_{k}$ are in different components of $\mathcal{A}_{p}$.

Let $\mathscr{B}_{1}$ be a cycle of length $n$. Then there exists $q \in P$ such that $q \geq p$ and $f^{n}\left(\varphi_{p q}\left(a_{i}\right)\right)=\varphi_{p q}\left(a_{i}\right)$ according to Lemma 3 and the definition of a direct limit. The element $\varphi_{p q}\left(a_{i}\right)$ belongs to an $n$-element cycle, since $\overline{\varphi_{p q}\left(a_{i}\right)}=u_{i}$ and $u_{i}$ belongs to an $n$-element cycle. In view of Lemma 1 elements $\varphi_{p q}\left(a_{1}\right), \ldots, \varphi_{p q}\left(a_{k}\right)$ are in different components of $\mathcal{A}_{q}$. Conclude that $\mathcal{A}$ contains $k$ cycles of the length $n$ as its components.

Now let $\mathscr{B}_{1}$ be a line. Let $i \in\{1, \ldots, k\}$. We get that $a_{i}$ is in no cycle according to the definition of a direct limit. Therefore the component of $\mathcal{A}_{p}$, which possesses $a_{i}$, is a line, since the operation of $\mathcal{A}_{p}$ is bijective. Conclude that $\mathcal{A}_{\text {contains }} k$ lines as its components.

We have proved that $\overline{\mathcal{A}}$ consists of at most such number of isomorphic components as $\mathcal{A}$.

Let $\mathcal{A}$ be an algebra without a cycle. Then every component of $\mathcal{A}$ is a line and $\overline{\mathcal{A}}$ is a retract of $\mathcal{A}$.

Suppose that $\mathcal{A}$ has a cycle. Let us order lenghts of cycles of the algebra $\mathcal{A}$ according to the divisibility and let minimal elements of this ordered set be $\left\{k_{i}, i \in I\right\}$. We remark that the set $I$ is nonempty and it is at most countable. Let $i \in I$. If $a$ belongs to a $k_{i}$-element cycle of $\mathcal{A}$, then every endomorphism of $\mathcal{A}$ maps $a$ into a cycle of length $k_{i}$. Thus $\bar{a}$ belongs to a cycle of length $k_{i}$ according to the direct limit definition. Therefore $\overline{\mathcal{A}}$ contains a cycle of length $k_{i}$ for every $i \in I$. That means that $\overline{\mathcal{A}}$ is a retract of $\mathcal{A}$.

Theorem 3. The number of all non-isomorphic monounary algebras with easy direct limits is equal to $2^{\mathbf{N}_{0}}$.

Proof. The number of all non-isomorphic monounary algebras with easy direct limits which have a bijective fundamental operation is equal to $2^{\boldsymbol{\aleph}_{0}}$ according to the previous theorem. Therefore the assertion is valid in view of Cor.2.

Proposition 4. The number of monounary algebras with easy direct limits which have $2^{\aleph_{0}}$ retracts is $2^{\aleph_{0}}$.

Proof. Let $K \subseteq \mathbb{N}$. Take an algebra $\mathcal{A}_{K}$ such that

(1) every component of $\mathcal{A}_{K}$ is a cycle,

(2) if $k \in K$, then $\mathcal{A}_{K}$ contains exactly 3 cycles of length $k$,

(3) if $k \notin K$, then $\mathcal{A}_{K}$ contains exactly 2 cycles of length $k$. 


\section{REFERENCES}

[1] Z. Farkasová and D. Jakubíková-Studenovská, "On radical classes of monounary algebras," Miskolc Math. Notes, vol. 14, no. 3, pp. 871-886, 2013.

[2] G. Grätzer, Universal algebra, 2nd ed. Springer, New York, 2008. doi: 10.1007/978-0-38777487-9.

[3] E. Halušková, "On iterated direct limits of a monounary algebra," in Contributions to general algebra, 10 (Klagenfurt, 1997). Heyn, Klagenfurt, 1998, pp. 189-195.

[4] E. Halušková, “Two element direct limit classes of monounary algebras," Math. Slovaca, vol. 52, no. 2, pp. 177-194, 2002.

[5] E. Halušková and M. Ploščica, "On direct limits of finite algebras," in Contributions to general algebra, 11 (Olomouc/Velké Karlovice, 1998). Heyn, Klagenfurt, 1999, pp. 101-103.

[6] J. Jakubík and G. Pringerová, "Direct limits of cyclically ordered groups," Czechoslovak Math. J., vol. 44(119), no. 2, pp. 231-250, 1994.

[7] D. Jakubíková-Studenovská and J. Pócs, "Cardinality of retracts of monounary algebras," Czechoslovak Math. J., vol. 58(133), no. 2, pp. 469-479, 2008, doi: 10.1007/s10587-008-0028-5.

[8] D. Jakubíková-Studenovská and J. Pócs, "On finite retract lattices of monounary algebras," Math. Slovaca, vol. 62, no. 2, pp. 187-200, 2012, doi: 10.2478/s12175-012-0003-3.

[9] D. Jakubíková-Studenovská and J. Pócs, "Some properties of retract lattices of monounary algebras," Math. Slovaca, vol. 62, no. 2, pp. 169-186, 2012, doi: 10.2478/s12175-012-0002-4.

[10] Jakubíková-Studenovská, D. and Pócs, J., Monounary algebras. P. J. Šafárik University in Košice, 2009.

[11] F. Krajník and P. Miroslav, "Compact intersection property and description of congruence lattices," Math. Slovaca, vol. 64, no. 3, pp. 643-664, 2014, doi: 10.2478/s12175-014-0231-9.

[12] J. Lihová, “On convexities of lattices," Publ. Math. Debrecen, vol. 72, no. 1-2, pp. 35-43, 2008.

[13] T. W. McKenzie R., McNulty G., Algebras, Lattices, Varieties, vol.1. Wadsworth, 1987.

[14] C. Pelea, "On the direct limit of a direct system of multialgebras," Discrete Mathematics, vol. 306, pp. 2916-2930, 2006.

[15] C. Pelea, "On convexities of lattices," Publ. Math. Debrecen, vol. 72, no. 1-2, pp. 35-43, 2008.

Author's address

Emília Halušková

Mathematical Institute, Slovak Academy of Sciences, Grešákova 6, 04001 Košice, Slovakia

E-mail address: ehaluska@saske.sk 